\title{
A Memetic Algorithm for Computing Multicriteria Shortest Paths in Stochastic Multimodal Networks
}

\author{
O. Dib \\ IRT SystemX, Paris-Saclay, \\ France \\ omar.dib@irt-systemx.fr
}

\author{
A. Caminada \\ Univ. Bourgogne Franche- \\ Comté, UTBM, France \\ alexandre.caminada@utbm.fr
}

\author{
M-A. Manier \\ Univ. Bourgogne Franche- \\ Comté, UTBM, France \\ marie-ange.manier@utbm.fr
}

\author{
L. Moalic \\ Univ. Bourgogne Franche- \\ Comté, UTBM, France \\ laurent.moalic@utbm.fr
}

\begin{abstract}
Modern transport systems are nowadays very complex. Building Advanced Travelers Information Systems (ATIS) has therefore become a certain need. Since passengers do not only seek a short-time travel, but they tend to optimize several criteria, an efficient routing system should incorporate a multiobjective analysis into its search process. Besides, the transport system may behave in an uncertain manner. Therefore, integrating uncertainty into routing algorithms may provide better itineraries. The main objective of this work is to propose a Memetic Approach (MA) in which a Genetic Algorithm (GA) is combined with a Hill Climbing (HC) local search procedure in order to solve the multicriteria shortest path problem in stochastic multimodal transport networks. Experimental results have been assessed by solving real life itinerary problems defined on the transport network of the city of Paris and its suburbs. Results indicate that unlike classical deterministic algorithms and pure GA and hill climbing, the proposed MA provide better itineraries within a reasonable amount of time.
\end{abstract}

\section{CCS CONCEPTS}

- Applied computing $\rightarrow$ Operations research; Transportation.

\section{KEYWORDS}

Multicriteria optimization, stochastic multimodal networks, genetic algorithms, hill climbing, memetic algorithms.

\section{INTRODUCTION AND MOTIVATIONS}

The demand for efficient routing methods that help passengers navigating through the intricate transport scheme has gained significant attentions in recent years. Companies and transport operators tend to build intelligent routing systems whereby passengers' routing queries are efficiently answered. Establishing such systems requires considering the various properties of the transport system and meeting the needs of

\footnotetext{
* Permission to make digital or hard copies of part or all of this work for personal or classroom use is granted without fee provided that copies are not made or distributed for profit or commercial advantage and that copies bear this notice and the full citation on the first page. Copyrights for third-party components of this work must be honored. For all other uses, contact the Owner/Author.

GECCO '17 Companion, July 15-19, 2017, Berlin, Germany

(C) 2017 Copyright is held by the owner/author(s).

ACM ISBN 978-1-4503-4939-0/17/07.

http://dx.doi.org/10.1145/3067695.3076064
}

passengers. To achieve that, several challenges have to be addressed. Firstly, an efficient representation for the transport system should be established in order to cope with basic and complex routing problems. Secondly, powerful routing algorithms should be designed in order to deal with routing issues.

The routing problem addressed in this work refers to solving the multicriteria shortest path problem in a stochastic multimodal transportation network [1]. The first difficulty to solve this problem lies in considering a multicriteria optimization problem. Indeed, in a multi-objective context, there is not only one single optimal solution, but rather a set of nondominated solutions, from which the decision maker must select his/her most preferred one [2]. Determining such Pareto set is a tedious task since one problem may have a huge number of nondominated solutions. The second difficulty originates in the presence of stochastic components in the problem, which represent travel times on edges. Stochastic travel times may be the result of many unanticipated events such as incidents and weather conditions.

The aforementioned difficulties will make the use of standard shortest path algorithms infeasible due to their high computational time and lack of flexibility to cope with stochastic changes. To overcome that, we propose in this paper a memetic algorithm to solve the emerging routing issue. MAs can be seen as an extension of GAs since they exploit a population based global search technique in order to identify promising search regions. In contrast with GAs, MAs use local search procedures in order to perform local refinements; thus, better search regions will be identified and probably better solutions will be obtained.

\section{PROPOSED MEMETIC ALGORITHM}

Before explaining the proposed MA, we first present how the transport system is represented. As a first step, two types of nodes, which correspond to stations and platforms are introduced. A station can encompass a set of platforms, whereas a platform cannot belong to more than one station. An edge may have one single weight if it is time independent or several weights if it is time dependent. To account for stochasticity, the travel time is considered as a random variable that varies according to a custom probability law.

After representing the multimodal network, we explain the proposed MA. As in standard GAs, the proposed approach proceeds with a set of initial solutions. These latters are generated using a constructive heuristic based on a double 
search algorithm [3]. The result of this operation is a set of initial feasible solutions to go from the departure to the arrival station with respect to the departure time.

To encode solutions, a vector of nodes where each node corresponds to a platform is used. This list represents a path from the departure to the arrival station. The length of a chromosome is variable and may not be greater than the number of platforms in the network. The fitness function of an individual is a 3-dimensional vector where each dimension represents one criterion (travel time, number of exchanges or walking time). After generating initial solutions, and in contrast to the standard GA's scheme, the initial population passes through an enhancement operation. That is, the Hill Climbing local search is applied over each individual in the initial population. By doing so, good initial individuals will be used for the evolution process.

To construct the list of neighboring solutions of a specific individual, the constructive heuristic used for generating initial solutions is applied. The best neighborhood is then used as a moving strategy. Deciding which solution is the best among all solutions in the neighborhood list is done by computing for each solution I in the neighborhood list, the number of solutions that I dominates in the list. The more a solution dominates other solutions, the better it is. After identifying the best neighbor solution $I^{*}$, the number of solutions dominated by the initial solution I0 is also computed. If $\mathrm{I}^{*}$ dominates more solutions than I0, then I* replaces I0. Otherwise, a local minimum is detected.

After enhancing initial solutions, the algorithm continues to perform some operations until a stopping criterion is met. To perform the selection, a stochastic roulette wheel is used where all chromosomes are placed according to their selection probability. Therefore, the better the chromosomes are, the more chances to be selected they have. Since several criteria are considered, a comparison mechanism is implemented to decide which chromosomes are the best in the current population. For this purpose, a rank is assigned to each individual according to its objectives' values. An individual is then better than another if its associated rank is higher. The rank of an individual represents the average value of all ranks determined by sorting all individuals in the population according to each objective.

The multiple point crossover has been used as a crossover operator. A crossover point is chosen to be a node where passengers exchange from one mode to another. After selecting two individuals, new individuals are produced with respect to the crossover points. By doing so, a new population having twice the size of the current population is produced. The best half individuals according to their global rank are then selected for the next generation and the rest are ignored.

To accomplish the mutation operation and in contrast to standard GAs, the hill climbing is used. That is, the local search is applied over survival individuals in order to enhance their quality. The algorithm stops when it fails to find interesting solutions during several steps. An interesting solution is a new generated individual that is not dominated by any individual in the current population or is a solution that at least dominates only one individual. Another stopping criterion is when the algorithm reaches a maximum number of generations.

\section{RESULTS AND DISCUSSION}

To evaluate this work, a routing application was developed based on the real data of the French region Île-de-France. The proposed MA is compared with: a) standard multicriteria labelsetting algorithm b) pure GA c) pure $\mathrm{HC}$ and a standard multicriteria algorithm that is applied a posteriori. Algorithms were tested on an Intel core I5 machine of 8 GB RAM.

The comparison is done by solving 1000 routing queries, each having a departure, arrival stations and a departure time uniformly generated at random. For each query, 1000 scenarios are considered. Instances are uniformly picked at random. Two indicators are used for comparisons a) the average running time and b) quality of solutions with respect to the multicriteria labelsetting algorithm applied a posteriori. The quality of solutions is computed by dividing the rank of each solution by the highest rank among all nondominated solutions in the Pareto set.

Results show that better itineraries are obtained using the proposed MA and the pure GA in comparison with the deterministic approach (applied a priori). While the average GAP to the optimality of the proposed MA does not exceed $3 \%$ and $8 \%$ when the pure GA is considered, the $\sim$ GAP of the deterministic approach may increase to $15 \%$.

When it comes to the Hill Climbing, results indicate that its average GAP to the optimality may reach $25 \%$ and $29 \%$ in the worst case. Therefore, it can be said that solutions provided when using the $\mathrm{HC}$ approach are very poor and not robust enough to cope with travel time changes.

When it comes to the evaluation of the running time performance of the various implemented approaches, results show that deterministic algorithms suffer from high computational time. The $\sim$ running time of the deterministic approaches may increase to 3 minutes and 5 minutes in the worst case. When it comes to heuristic approaches, results indicate that the $\sim$ running time of the proposed MA does not exceed 150 milliseconds while it decreases to 135 milliseconds for the pure GA and to 120 for the Hill climbing.

The high computational time of the proposed MA in comparison with the GA and $\mathrm{HC}$ can be explained by the fact that MA requires additional time to enhance initial solutions and to perform the mutation via the local search. The low computational time of the $\mathrm{HC}$ can be explained by the fact the approach rapidly converges to a local minimum.

Finally, we focus in this work on solving the multicriteria shortest path problem in a stochastic network; Experimentations proved the efficiency of the proposed MA. Integrating other transport modes such as bike is planned to be done in the future.

\section{REFERENCES}

[1] Rajabi-Bahaabadi, Mojtaba, et al. "Multi-objective path finding in stochastic time-dependent road networks using non-dominated sorting genetic algorithm." ESWA 42.12 (2015): 5056-5064.

[2] Dib, Omar, Marie-Ange Manier, and Laurent Moalic. "Advanced modeling approach for computing multicriteria shortest paths in multimodal transportation networks." Intelligent Transportation Engineering (ICITE), IEEE International Conference on. IEEE, 2016.

[3] Dib, O., et al. "An advanced GA-VNS combination for multicriteria route planning in public transit networks." Expert Systems with Applications 72 (2017): 67-82 